\title{
Iris Recognition Algorithm based on MMC-SPP
}

\author{
Yongqiang LI \\ Software Technology Vocational College, North China University of Water \\ Resources and Electric Power \\ Zhengzhou 450045, China \\ 1696450309@qq.com
}

\begin{abstract}
Sub patter algorithm does not consider the structure relationship between the same sample in different modes, it is difficult to accurately reveal the space features of the iris image, this paper proposes a iris recognition algorithm based on maximum margin criterion(MMC) and structure preserving projection (SPP). Firstly, the original high dimensional data is divided into several subsystems, and then SPP algorithm is used to reduce the iris feature dimension, the structural relationship between iris sample pattern collection manifold structure and the same sample of different pattern at the same time, finally, MMC function is introduced to improve the ability of classification algorithm by considering the iris category information, and the nearest neighbor classification algorithm is used to classify the iris image, and the recognition result of iris images is obtained by using the voting method. The performance of the algorithm is tested on CASIA 1.0 and CASIA 2.0 iris database, and the results show that the proposed algorithm has improved the recognition rate and recognition speed of iris image compared with the other algorithms.
\end{abstract}

Keywords : iris recognition, structure supervised preserving projection, maximum margin criterion, sub patter

\section{Introduction}

As an important biological feature, iris has the unique, collectable and non-invasive advantages. Based on the iris identification, it has been widely applied in many fields. Accurate and fast iris recognition algorithm thus becomes a heatedly discussed topic in the current pattern recognition field [1].

In view of iris recognition issue, domestic and overseas scholars have done a lot of in-depth researches, and put forward many effective iris recognition methods. Iris image has high dimensionality, and its feature dimensions often exceed the number of samples, so that the iris image is sparse in high-dimensional spatial distribution. If the original features of iris image are directly entered into the classifier for learning, in which the useless and redundant features adversely affect the iris image' recognition rate, resulting in low identify efficiency [2]. In order to solve the "curse of dimensionality" and small sample size issues incurred by iris's high-dimensional feature and improve the iris recognition rate and efficiency, some scholars have proposed the sub-mode-based iris recognition algorithm [3]. Sub-mode-based iris recognition algorithm refers to project the high-dimensional iris image onto the low-dimensional subspace by adopting certain dimensionality reduction methods, eliminate the useless and redundant features, and extract the iris features in low-dimensional spaces. Current iris feature dimensionality reduction techniques consists of the Principal Component Analysis (PCA), Independent Component Analysis (ICA), Linear Discriminant Analysis (LDA), Isometric Feature Mapping (ISOMAP), Locally Linear Embedding algorithm (LLE), Laplacian Eigenmap algorithm (LE), Locality Preserving Projection (LPP) and so on [4-7]. PCA, ICA, LDA 
are a class of linear dimension reduction method, which can only extract the global low-dimensional features. It is difficult to find the nonlinear manifold structure embedded in the iris's local sub-mode set [8]. ISOMAP, LLE, LE, LPP belong to the manifold learning algorithm, which has advantages in maintaining the iris's nonlinear manifold structure [9-11]. However, LE, LPP and other algorithms can only retain the iris image's popular structure without consideration of the structural relationship between all sub-modes, so they are unable to fully and accurately describe the category information of the iris, limiting the application scope of the iris recognition [12].

To further improve the iris recognition rate and efficiency, this paper proposes a structure preserving projection (SPP) iris feature extraction algorithm, and integrates it with the Maximum Margin Criterion (MMC) to construct a new iris recognition method (MMC-SPP). In the SPP algorithm iris feature process, this paper maintains structural relationships between the same iris samples and different sub-modes as well as the manifold structure of iris sample sub-mode set, considers the class information of the iris data, introduces MMC function, improves SPP algorithm's classification performance, and finally verifies the effectiveness of MMC-SPP algorithm by using CASIA 1.0, 2.0 iris data set. The results show that MMC-SPP algorithm can effectively eliminate the useless and redundant features among high-dimensional iris features, extract the iris feature having the greatest contribution to the recognition results, and it is an iris recognition algorithm with high recognition rate and fast speed.

\section{MMC Algorithm}

MMC is a subspace supervised learning method. It has improved the LDA algorithm, converts the quotient-based objective function into difference-based objective function, solves the small sample size problem of LDA, avoids the LDA operation towards the matrix inversion, and reduced the calculation amount ${ }^{[13]}$. MMC algorithm's objective function is:

$$
J=\max \left\{\sum_{i, j} p_{i} p_{j}\left(d\left(m_{i}, m_{j}\right)-\operatorname{tr}\left(S_{i}\right)-\operatorname{tr}\left(S_{j}\right)\right)\right\}
$$

Where, $m_{\mathrm{i}}$ is the average of the $\mathrm{i}$-th data, $p_{i}$ is the prior probability, $d\left(m_{i}, m_{j}\right)$ is the Euclidean distance between the i-th and j-th data, and $S_{i}$ is the intra-class scatter matrix.

Equation (1) is to maximize the distance between the data with different classes, the below equation can be obtained through a series of derivation:

$$
J=\mathrm{m} \text { a } \mathrm{x} r \mathrm{~S}_{b}-S_{w}
$$

Where, $S_{b}$ represents the inter-class scatter matrix; and $S_{w}$ stands for intra-class scatter matrix.

Assumed that there are $c$ class data and the data set is $m$, the following equations are obtained

$$
\begin{aligned}
S_{b} & =\sum_{i=}^{c} p_{i}\left(m_{i}-m\right)\left(m_{i}-m\right)^{T} \\
S_{w} & =\sum_{i=}^{c} p_{i} S_{i}
\end{aligned}
$$

Introduce $\beta$ to weigh $S_{b}$ and $S_{w}$, and the objective function of MMC algorithm is:

$$
J=\max \operatorname{tr}\left(S_{b}-\beta S_{w}\right)
$$

\section{MMC-SPP Iris Recognition Algorithm}

MMC-SPP Iris Recognition Algorithm is a sub-mode-based recognition algorithm, and it consists of the following steps: (1) Divide the sub-modes of iris images; (2) Use SPP 
algorithm to reduce the dimension of sub-modes and extract iris features; (3) Recognize the unknown iris images.

\subsection{Sub-mode Division of Iris Images}

Assumed the training set consists of $N_{p}$ individual $N$ pieces of iris images: $X=\left[x_{1}\right.$, $\left.x_{2}, \ldots, x_{N}\right]$, its pixel size is $H_{1} \times H_{2}$, uses matrix partitioning method to divide each iris image into $C$ sub-images with equal size, and then convert them into dimensions of vector: $H 1 \times H 2 / C$. When all training samples are divided into sub-images, sub-modes of different images will be composed of $C$ sub-mode sets [14]. Iris image sub-mode set is as shown in Figure 1.

\section{Figure 1. Sub-mode Construction of Iris Image}

\subsection{Iris Feature Dimensionality Reduction of SPP Algorithm}

Assumed that the cth sub-mode of the $\mathrm{i}$-th iris training sample is $x_{i}^{c}(c=1,2, \ldots, C$; $i=1,2, \ldots, N)$, and the overall objective of SPP algorithm to extract the iris feature is: to find a projection matrix $W$, project $x_{i}^{c}$ onto the low-dimensional space, and then $y_{i}^{c}=W^{T} x_{i}^{c}$ is the dimensionality reduction results of $x_{i}^{c}$.

\subsubsection{Maintain the Structural Relationship Between the Same Iris's Each Sub-mode}

The first objective of SPP algorithm is to keep the structural relationship between the same iris image's each sub-mode unchanged. Given that the i-th iris training sample's $c$ th sub-mode set is: $X^{i}=\left[x_{1}^{i}, x_{2}{ }^{i}, \ldots x_{c}^{i}\right]$, then the first objective can be described as:

$$
E=\sum_{i=1}^{c}\left\|x_{i}^{c}-\sum_{l \in(-c)} a_{i}^{c l} x_{i}^{l}\right\| \|^{2}
$$

Where, $E$ is the reconstruction error, $a_{i}^{c l}$ is the $l$-th sub-mode's reconstruction coefficient towards the $c$-th sub-mode.

The reconstruction error of $x_{i}^{c}$ is:

$$
\varepsilon=\sum_{l \in(-c)} \sum_{m \in(-c)} a_{i}^{c l} a_{i}^{c m} G_{l m}
$$

Where, $G_{l m}=\left(x_{i}^{c}-x_{i}^{l}\right)^{T}\left(x_{i}^{c}-x_{i}^{m}\right)$.

Through the least square, the reconstruction coefficient is calculated as:

$$
a_{i}^{c l}=\frac{\sum_{m \in\{-c\}} G_{l m}^{-1}}{\sum_{p \in\{-c\}} \sum_{q \in\{-c\}} G_{p q}^{-1}}
$$

To remain each training sample's structural relationship unchanged in low-dimensional space, assumed that the reconstruction coefficient of $x_{i}^{c}$ can be expressed as $y_{i}^{c}$ in a low-dimensional subspace. To get a low-dimensional feature of local sub-image, it is necessary to minimize the iris image, namely

$$
\varepsilon=\left\|y_{i}^{c}-\sum_{l \in(-c)} a_{i}^{c l} y_{i}^{l}\right\|^{2}
$$

The objective functions of all $N$ pieces of image with concentrated training: 


$$
\begin{aligned}
& \min \sum_{i=1}^{N} \sum_{c=1}^{C}\left\|y_{i}^{c}-\sum_{l \in(-c)} a_{i}^{c l} y_{i}^{l}\right\|^{2} \\
& =\min \sum_{i=1}^{N} \sum_{c=1}^{C}\left\|W^{T} x_{i}^{c}-\sum_{l \in(-c)} a_{i}^{c l} W^{T} x_{i}^{l}\right\|^{2}
\end{aligned}
$$

Given $P=\sum_{i=1}^{N} X_{i} M_{i} X_{i}^{T}$, Equation (10) can be simplified as:

$$
\min \operatorname{tr}\left(W^{T} Q W\right)
$$

\subsubsection{Maintain the Manifold Structure for Sub-mode with Different Iris's Same Position}

The second objective of SPP algorithm is to keep different iris images in the sub-mode manifold structure with the same position. Assumed that the sub-mode set of all images' $c$-th sub-mode is: $X^{c}=\left[x_{1}{ }^{c}, x_{2}{ }^{c}, \ldots, x_{N}{ }^{c}\right]$, the corresponding subspace features are: $Y^{c}=\left[y_{1}{ }^{c}, y_{2}{ }^{c}, \ldots, y_{1}{ }^{c}\right]$. The second objective can be realized by keeping neighbor relationship between data points, namely, the below cost function is to be minimized:

$$
\begin{aligned}
& \min \sum_{i=1}^{N} \sum_{j=1}^{N}\left\|y_{i}^{c}-y_{j}^{c}\right\| S_{i j}^{c} \\
& =\min \sum_{i=1}^{N} \sum_{j=1}^{N}\left\|W^{T} x_{i}^{c}-W^{T} x_{j}^{c}\right\| S_{i j}^{c}
\end{aligned}
$$

Where, $S_{i j}^{c}$ is the similarity of $x_{i}^{c}$ and $x_{j}^{c}$ in high-dimensional space, which is defined as below:

$$
S_{i j}^{c}=\left\{\begin{array}{cc}
\exp \left(\frac{-\left\|x_{i}^{c}-x_{j}^{c}\right\|}{t}\right), x_{i}^{c} \in N\left(x_{j}^{c}\right) \text { or } x_{j}^{c} \in N\left(x_{i}^{c}\right) \\
0, & \text { others }
\end{array}\right.
$$

In the sub-image construction stage, the training set's iris image is divided into $C$ sub-mode sets. For all sub-mode sets, the objective function of manifold structure's SPP algorithm is:

$$
\begin{aligned}
& \min \sum_{c=1}^{C} \sum_{i=1}^{N} \sum_{j=1}^{N}\left\|y_{i}^{c}-y_{j}^{c}\right\| S_{i j}^{c} \\
& =\min \sum_{c=1}^{C} \sum_{i=1}^{N} \sum_{j=1}^{N}\left\|W^{T} x_{i}^{c}-W^{T} x_{j}^{c}\right\| S_{i j}^{c} \\
& =\min \sum_{i=1}^{C} \operatorname{tr}\left(W^{T} X^{c} L^{c} X^{c^{T}} W\right)
\end{aligned}
$$

Given $P=\sum_{i=1}^{C} X^{c} L^{c} X^{c^{T}}$, Equation (14) is:

$$
\min \operatorname{tr}\left(W^{T} P W\right)
$$

Finally, integrate the objective function (11) and (15), SPP algorithm's overall objective function can be expressed as:

$$
\min \operatorname{tr}\left\{W^{T}(P+\theta Q) W\right\}
$$

Where, $\theta$ is the weigh parameter between two objective functions, and meets $\theta \geq 0$ The corresponding constraint condition is:

$$
\left\{\begin{array}{l}
W^{T} Z Z^{T} W=I \\
Z=\left[x_{1}^{1}, x_{1}^{2}, \ldots x_{1}^{C}, \ldots, x_{N}^{1}, \ldots x_{N}^{C}\right]
\end{array}\right.
$$




\subsubsection{Overall Objective Function of MMC-SPP Algorithm}

In view of the feature space's the identification performance, introduce the maximum interval criterion function (5) in the SPP algorithm, use the sample's class information to improve the classification performance of the algorithm, and obtain the below overall objective function of SPP algorithm:

$$
\left\{\begin{array}{l}
\arg \min _{W} \operatorname{tr}\left\{W^{T}\left(P+\theta Q-S_{b}+\beta S_{w}\right) W\right\} \\
\text { s.t. } \quad W^{T} Z Z^{T} W=I
\end{array}\right.
$$

By using Lagrange multiplier method, the optimization problem (18) can be transformed into the below generalized feature decomposition problem:

$$
\left(P+\theta Q-S_{b}+\beta S_{w}\right) W=\lambda Z Z^{T} W
$$

Divide the above equation's eigenvalues, and take the minimum $d$-th eigenvalue's corresponding eigenvector to build the mapping matrix:

$$
W=\left[w_{1}, w_{2}, \ldots, w_{d}\right]
$$

\subsection{Iris Recognition}

For unknown iris image, first divide it into $c$ non-overlapping sub-modes: $U=\left[\begin{array}{lll}u^{1}, & u^{2}, \ldots, u^{c}\end{array}\right]$, then its low-dimensional subspace is featured by: $V=W^{T} U=\left[v^{1}, v^{2}, \ldots, v^{c}\right]$. For $v^{c}(c=1,2, \ldots$, c) , the Euclidean distance matrix between all low-dimensional features of the $c$-th sub-mode set in the training set is:

$$
D M^{c}=\left[d\left(v^{c}, y_{1}^{c}\right), d\left(v^{c}, y_{2}^{c}\right), \ldots d\left(v^{c}, y_{N}^{c}\right)\right]
$$

The $c$-th sub-mode sample in the training set comes from different iris images, and classify by using the nearest neighbor classification algorithm. Since $V$ has c sub-modes, the final classification of iris image recognition can be obtained by voting method, and the probability of unknown iris belonging to $j$-th person is

$$
p_{j}=\frac{1}{C} \sum_{i=1}^{C} q_{i}^{j}
$$

Where, $q_{i}^{j}=\left\{\begin{array}{l}1, \text { if the }{ }^{i} \text { th subpattern is classfied to the }{ }^{j} \text { th person } \\ 0, \quad \text { otherwise }\end{array}\right.$

The final recognition result of unknown iris is

$$
\operatorname{Identity}(u)=\arg \max \left(p_{j}\right), j=1,2, \ldots, N_{p}
$$

\section{Experimental Results and Analysis}

\subsection{Data Source}

To verify the effectiveness and superiority of the MMC-SPP algorithm, this paper selects the iris images from the iris database of Institute of Automation, Chinese Academy of Sciences - CASIA 1.0 and CASIA 2.0 as the test object, and part of the iris images are shown in Figure 2 [15]. To test the MMC-SPP algorithm's superiority over iris recognition, the paper adopts three feature dimensionality reduction methods (PCA, LLE, LPP) for feature dimensionality reduction and extraction comparative experiments.

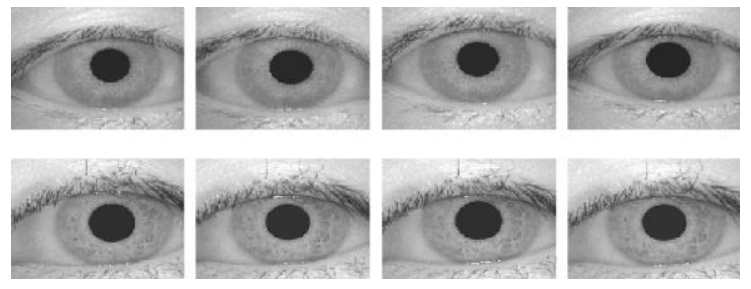




\section{Figure 2. Part of Iris Images}

\subsection{CASIA 1.0 Database}

\subsubsection{Recognition Rate Comparison}

In CASIA 1.0 iris database, the image size is $280 \times 320$ pixels with iris images for 108 eyes, 7 images for each eye and a total of 756 images. It uses contacted device to shoot, which is less affected by the light and eyelash blocking. Randomly select 5 images as the training set for each eye, and the rest 2 images are used as the test set. To facilitate processing, the iris image is adjusted to $100 \times 100$ pixels. MMC-SPP algorithm parameters are set as: neighborhood parameter $k=5$, thermonuclear parameters $t=100, \theta=0.8$. In LPP algorithm, $t=10$. First, convert each iris image into 25 sub-modes, that is, the size of each sub-mode is set to be $20 \times 20$. The change relationship between the recognition rate and dimensions of each iris feature extraction algorithm is shown in Figure 3. According to Figure 3, MMC-SPP algorithm has comprehensively taken into account of the structural relationship between each sub-mode in the same iris and the manifold structure of sub-mode set. When extracting the iris features, the iris class information can be described in a more accurate and comprehensive manner. It thus improves the iris recognition performance, and the average recognition rate is up to $93.65 \%$, much higher than $91.01 \%$ of PCA algorithm, $88.9 \%$ of LLE algorithm, $88.56 \%$ of LPP algorithm, indicating that the proposed iris recognition idea is correct.

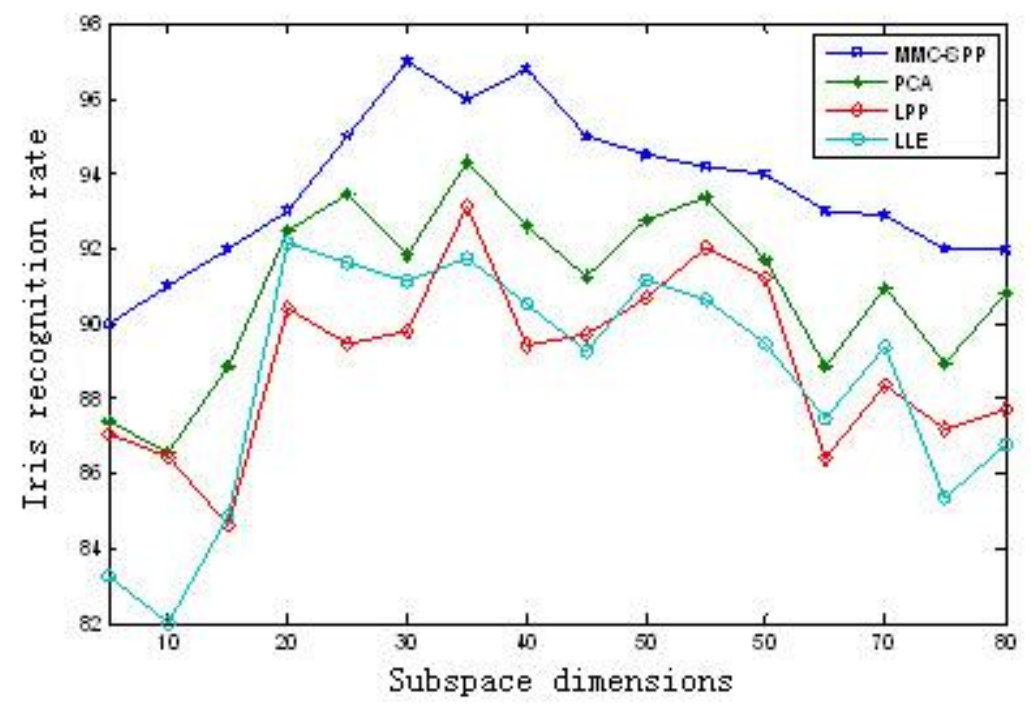

Figure 3. Recognition Rate Comparison of Different Algorithms in CASIA 1.0 Database

\subsubsection{Comparison of Recognition Speed}

The recognition speed is very important for the real-time iris recognition, and the time (seconds, s) is used as the evaluation criteria of iris recognition speed. On Core2 Intel 2.8GHZ CPU, RAM 2.0G, Windows XP platform, use tic and toc commands of Matlab 2012 to record each algorithm's average training and recognition time for CASIA 1.0 database, and the results are shown in Table 1. According to Table 1, compared to the comparison algorithm, MMC-SPP is featured by the shortest running time, the fastest recognition speed and the highest recognition efficiency. This is because MCC-SPP algorithm can find optimal iris feature, effectively reduce the feature dimensions, and eliminate a lot of useless, redundant features, so that MCC-SPP can realize the real-time iris recognition. 
Table 1. Speed Comparison of Different Algorithms

\begin{tabular}{ccc}
\hline Model & Training time(s) & Test time(s) \\
\hline PCA & 6.75 & 0.88 \\
LPP & 5.18 & 0.77 \\
LLE & 5.03 & 0.59 \\
MMC-SPP & 4.99 & 0.56 \\
\hline
\end{tabular}

\subsubsection{Subspace Dimensions for Different Feature Extraction Algorithms}

For different algorithms' optimal recognition rate and the corresponding subspace dimension, please refer to Table 2. According to Table 2, although the subspace dimensions of LLE, LPP is similar to SPP algorithm, LLE and LPP has too low recognition rate, which cannot meet the accuracy requirements of iris recognition. MMC-SPP has the highest recognition rate, reaching $97.00 \%$, at least $2 \%$ higher than PCA, LLE and LLP algorithm.

Table 2. Iris Recognition Results of CASIA 1.0

\begin{tabular}{ccc}
\hline Iris Feature Extraction Algorithm & Subspace Dimensions & Recognition Rate $(\%)$ \\
\hline PCA & 35 & $94.30 \%$ \\
LPP & 20 & $93.12 \%$ \\
LLE & 20 & $92.17 \%$ \\
MMC-SPP & 20 & $97.00 \%$ \\
\hline
\end{tabular}

\subsubsection{Effects of Training Sample Size on Recognition Rate}

The changing curve of recognition rate effects between training samples with different proportions and testing samples is shown in Figure 4. From Figure 5, when there is less training samples, all iris recognition algorithms are low. As the number of training samples increases, the recognition rate also increases. When selecting 5 images from MMC-SPP algorithm as the training samples, the iris recognition rate is up to $97 \%$ and the recognition rate is significantly superior to the comparison algorithm.

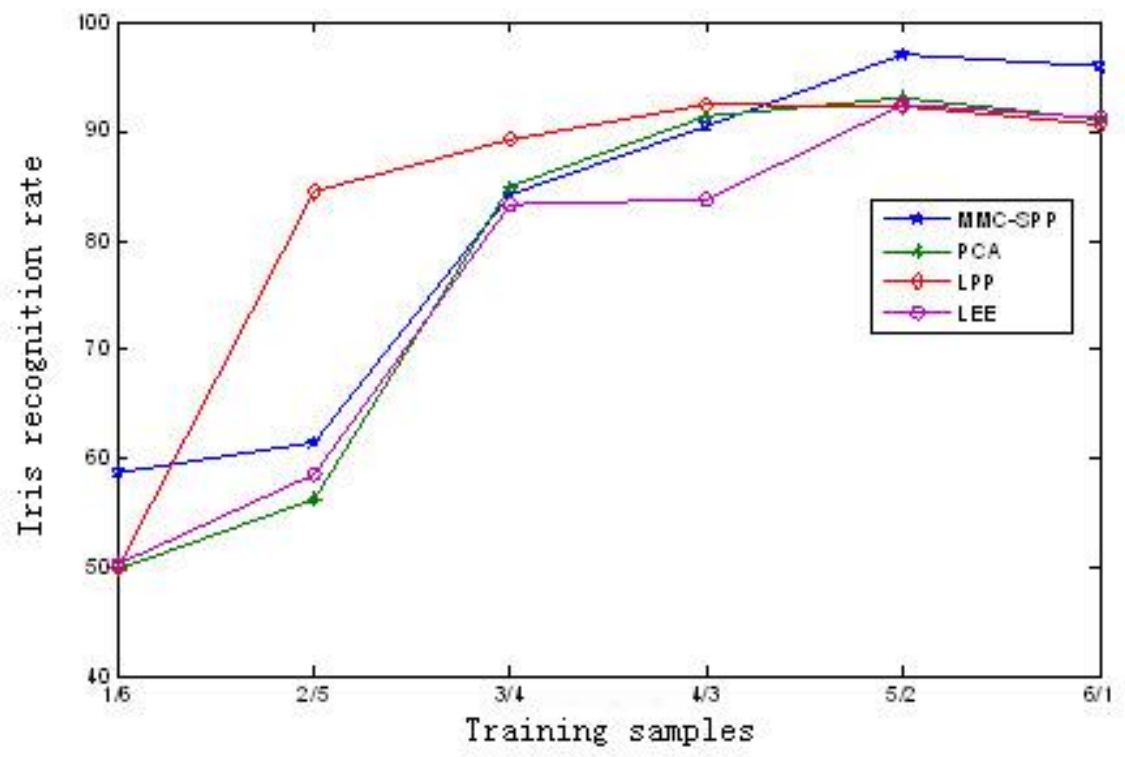

Figure 4. Changing Curve between the Number of Training Sample and Iris Recognition Rate 


\subsection{Comparison with Classical Algorithm}

To make the results of MMC-SPP algorithm more convincing, this paper takes the current classical iris recognition algorithm to conduct comparative experiment towards CASIA 1.0, 2.0 iris database set under the same conditions, and the average iris recognition rate is as shown in Figure 7. According to Figure 5, MMC-SPP's average iris recognition rate is higher than the classical iris recognition algorithm. The comparative results show that MMC-SPP is a fast and efficient iris recognition algorithm with high recognition rate.

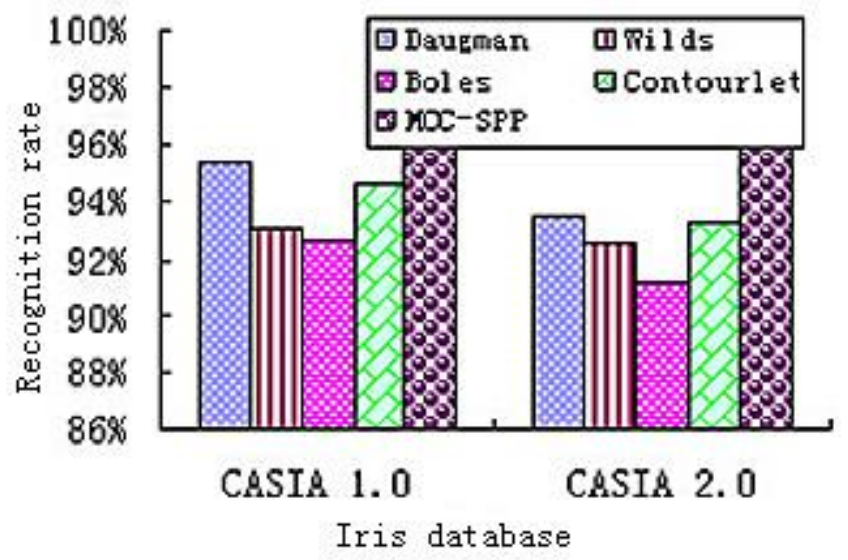

Figure 5. Performance Comparisons with Classical Iris Recognition Algorithm

\section{Conclusion}

For feature extraction issues in the iris recognition, this paper presents an iris recognition algorithm based on MMC-SPP. MMC-SPP algorithm reduces the dimension of all sub-mode iris images to the uniform low-dimensional subspace, and maintains the global structural information and manifold structure of local iris sub-mode, so as to greatly retain the high-dimensional data's internal structure, introduce the MMC improvement algorithm classification ability with supervised learning ability, and verify the effectiveness and superiority of MMC-SPP algorithm through two standard iris data sets.

\section{Acknowledgments}

Thanks for the CASIA iris database provided by Institute of Automation, Chinese Academy of Sciences, so that the present study can be made smoothly.

\section{References}

[1]. Z. F. He, T. N. Tan, Z. N. Sun and X. C. Qiu, "Towards Accurate and Fast Iris Segmentation for Iris Biometrics", IEEE Trans. Pattern Analysis and Machine Intelligence, vol. 31, no. 9, (2009), pp. 1670-1684.

[2]. Z. N. Sun and T. N. Tan, "Ordinal Measures for Iris Recognition”, IEEE Trans. Pattern Analysis and Machine Intelligence, vol. 31, no. 12, (2009), pp. 2211-2226.

[3]. Z. He and L. Lv, "Iris feature extraction and recognition based on ICA-MJE and SVM", Computer Applications, vol. 27, no. 6, (2007), pp. 1505-1507.

[4]. Y. Huang, S. Luo and E. Chen, "Iris Recognition Method based on Independent Component Analysis", Computer Research and Development, vol. 40, no. 10, (2003), pp. 1451-1457.

[5]. N. Sun, W. Yu and M. Cao, "Iris Recognition Method based on PCA and ICA", Chinese Journal of Image and Graphics, vol. 13, no. 9, (2008), pp. 1701-1706.

[6]. M. Xie and S. Shao, "Iris Recognition Based on feature Extraction in Kernel Space", Proceeding of international Conference on Communications, Circuits and Systems, Kokura, (2007), pp. 760-76. 
[7]. Z. Luo and S. Lin, "Iris Image Preprocessing Method for Identity Authentication", South China University of Technology (Natural Science Version), vol. 37, no. 5, (2009), pp. 43-48.

[8]. A. Engin, S. Abdukadir and H. Davut, "An optimum feature extraction method for texture classification", Expert System with Applications, vol. 36, no. 3, (2009), part2:6036-6043.

[9]. X. Song and S. Ye, "Manifol Learning Algorithm based on Local Linear Approximation", Computer Simulation, vol. 25, no. 7, (2008), pp. 86-89.

[10]. J. Wang Jianguo, W. Yang and J. Yang, "Maximum Scatter Difference Feature Extraction Method based on Preserving Projection", Mode Recognition and Artificial Intelligence, vol. 22, no. 4, (2009), pp. 610-613.

[11]. J. Wei and P. Hong, "Semi-supervised Dimensionality Reduction Method based on Local and Global Preservation", Journal of Software, vol. 19, no. 11, (2008), pp. 2833-2842.

[12]. X. F. He and G. -y. Ni, "Locality preserving projections", Advance in Neural Information Processing Systems, vol. 16, (2004), pp. 153-160.

[13]. X. Qu, M. He and S. Mei, "An Improved Locality Preserving Projection Hyperspectral Feature Extraction Algorithm", Modern Electronics Technique, vol. 34, no. 13, (2011), pp. 74-77.

[14]. X. Wang and Q. Dong, "An Iris Recognition Algorithm based on 2D-PLDA and Wavelet Sub-band", Chinese Journal of Image and Graphics, vol. 16, no. 1, (2011), pp. 59-64.

[15]. Institute of Automation, Chinese Academy of Sciences. CASIA Iris Image Database [DB/OL]. (2003-10)[2005-08]. http://www.cbsr. ia.ac.cn/IrisDatabase/irislogin.html.

\section{Author}

Li Yongqiang (1974.1-) Lecturer, Master, Research Orientation: Computing Network 
International Journal of Signal Processing, Image Processing and Pattern Recognition Vol. 8, No. 2 (2015) 